\title{
Effect of initial tangential intensity on the fluid dynamic characteristics in tangential burner
}

\author{
Pasymi $^{1,2}$, Yogi Wibisono Budhi ${ }^{1}$, and Yazid Bindar ${ }^{*}$ \\ ${ }^{1}$ Department of Chemical Engineering, Faculty of Industrial Technology Institut Teknologi Bandung, Bandung, Indonesia \\ ${ }^{2}$ Department of Chemical Engineering, Faculty of Industrial Technology Bung Hatta University, Padang, Indonesia
}

\begin{abstract}
Swirl turbulent flow is intensively used by the industrial equipments such as combustion, separation and heat transfer equipments. The fluid dynamic characteristics of this flow are influenced by the chamber's geometries and the operating conditions. One of the important operating condition variables which greatly affects the fluid dynamic characteristics is initial tangential intensity (IтI) or often known as swirl number. This study is aimed to quantify the effect of the initial tangential intensity on the fluid dynamic characteristics in a tangential burner. The method of the study is based on the computational fluid dynamic simulation under the Ansys Fluent CFD engine. The fluid dynamic characteristics were modeled using the standard $k-\varepsilon$ turbulent model. The simulation results exhibited that the three dimensional flow structure in a tangential burner is dominated by the tangential flow. The fluid dynamic simulations also showed that the effect of the $\mathrm{I}_{\text {TI }}$ on the mean turbulence intensity and the mean residence time begin to be significant at the $\mathrm{I}_{\mathrm{TI}}$ values $\geq 1.1$ and $\geq$ 4.5 respectively, while at low ITI values, its effects on both variables are insignificant. The lowest pressure drop obtained in this study was found on the burner with ITI value of 3.2.
\end{abstract}

\section{Introduction}

A tangential burner is a burner that has two inflow orientations, namely axial orientation and tangential orientation. The tangential inflow orientation can be created through a tangential injection or a swirler. This burner type has several advantages over the axial burners. These advantages include increasing the degree of the mixing, extending the residence time of fuel in the burner and assisting the ash separation.

Initially, the development of this burner was intended to reduce coal ash problems in the boiler furnace. But now, a tangential burner is developed for the combustion of other solid fuels, such as biomass fuels. The tangential burners for a solid biomass fuel were developed previously by the names of the McConnell-48 burner [1], the VTS burner [2], and the baggase burner [3].

The performances of a tangential burner are greatly influenced by the burner geometry factors, and the operating conditions. The geometry factors include the shape and the size of the chamber, and the shape and the size the inlet and the outlet. The operating conditions cover the variables of the total mass flow rate and the initial tangential intensity ( $\left.\mathrm{I}_{\mathrm{TI}}\right)$.

The initial tangential intensity is defined as the ratio between the mass flux entering the tangential inlet to the mass flux entering the burner cylinder.
Mathematically, the initial tangential intensity of the flow in the tangential burner is evaluated using the following formula,

$$
I_{I T}=\left(A_{c} / A_{t}\right) \dot{m}_{t}^{2} /\left(\dot{m}_{t}+\dot{m}_{c}\right)^{2}
$$

The variables $A_{t}$ and $A_{c}$ are the cross-sectional area of the tangential inlet and burner cylinder, respectively. While the variables $\dot{m}_{t}$ dan $\dot{m}_{c}$ are the mass flow rate through the tangential inlet and the mass flow rate through the burner cylinder [4].

This study is aimed to quantify the effect of the initial tangential intensity to the flow characteristics in a tangential burner. The flow characteristics quantified here are the residence time, the turbulence intensity, the flow structure and the pressure drop. All these flow characteristics are well quantified three dimensionally by CFD display techniques.

\subsection{Swirl flow}

The swirl turbulent flow is dominated by the tangential velocity component. This typical turbulent flow has many uses in the industrial practices. Therefore, the studies related to this topic obtain great attention from researchers. Both, experimental and numerical methods are commonly used.

Bourgouin [5] conducted the study experimentally and numerically about the influence

\footnotetext{
* Corresponding author at yazid@che.itb.ac.id
} 
of a swirler geometry on the flow structures inside a gas turbine chamber using the LES approach. They claimed that their predictions agree with the corresponding experimental data. They stated that the flow structures and the precessing vortex core (PVC) frequency are extremely sensitive to the swirler geometry and have a strong effect on the combustion performances [5].

A simulation study on the ability of Unsteady Reynolds Averaged Navier-Stokes (URANS) approach to predict the fluid dynamics inside the swirl burner was reported by Vazquez [6]. He used both $k-\varepsilon$ and RSM turbulent models. He reported that both models can predict the values close to the experimental data. For an atmospheric swirl burner, the predictions with $k-\varepsilon$ model for the axial and the tangential velocity are better than the RSM model [6].

Escue and Cui [7] investigated the performance of several RANS turbulent models in modeling the developing swirl turbulent flow inside a straight pipe. They reported that although RANS models do not adequate in simulating the developing swirl turbulent flow, but they exhibit a better agreement with the experimental velocity profiles, especially for the low swirl flows. The RSM model even gave an appropriate prediction for higher swirl flows [7].

Another study concerning the flow structure and the combustion performances inside a swirl burner was conducted by Nemoda [8]. They used $k-\varepsilon$ turbulent model. Their simulation results satisfactorily agreed with the experimental data. They stated that the flow structures in the swirl burner are influenced by the swirl number or $\mathrm{I}_{\mathrm{TI}}$ [8].

\subsection{Fluid dynamics characteristics}

\subsubsection{Turbulence intensity}

The degree of the mixing is one of the variables that affects the combustion performances. The degree of the mixing inside a chamber can be indicated by the turbulence intensity. It can be determined experimentally or numerically.

According to Nemoda et al. (2005), the flow structure in a burner is influenced by the swirl number. At a high swirl number $(\mathrm{S} \geq 2.48)$, the recirculation flow structure exists and tends to increase the chemical conversion and the flame stability [8]. In line with the Nemoda results, Li et al. [9] reveals that the turbulence intensity value influences the combustion process and the $\mathrm{NO}_{\mathrm{x}}$ formation in a burner [9].

\subsubsection{Residence time}

The particles movement in the burner can be considered being similar to the air movement. Therefore, the air fluid dynamic in the burner can be used to estimate the pseudo particle residence time.
The residence time of the particles inside the burner is always associated with the particle burning time and the combustion degree that is expected to occur in the burner, whether a partial combustion or a complete combustion. The particle burning time is influenced by the degree of the mixing, the particle shape and size, the oxygen concentration and the temperature. Normally, the greater the particle burning time, the longer the residence time is required.

Momeni et al. [10] conducted the experiments for a combustion process of a single biomass particle in a combustion chamber. Their results showed that the particle combustion process is influenced by the shape and the size of the particle (the particle aspect ratio), the oxygen concentration and the temperature. For a biomass particle with the particle aspect ratio 4 , the oxygen concentration $20 \%$, and the burner temperature $1200{ }^{\circ} \mathrm{C}$, the burnout time of the particle was found at 5.4 seconds, while for the burner temperature $1600{ }^{\circ} \mathrm{C}$, the burnout time was found at 3.6 seconds [10].

\subsubsection{Pressure drop}

A pressure drop across the separation and the combustion chamber is directly related to the operating cost. The higher the pressure drop, the higher the cost to drive the carrier fluid across the chamber is. The swirl flow is known to increase the pressure drop in the chamber.

Aydin et. al [11] investigated experimentally the efect of the swirler geometry, the Reynolds number and the cylinder ratio on the pressure drop and the heat transfer performance. They stated that all of the above variables have the effects on the pressure drop and the heat transfer. Unlike the effect of the swirler geometry and the cylinder ratio on the pressure drop which can be ignored, the effect of the Reynolds number on the pressure drop is so significant [11].

\subsection{Turbulent model}

There are three advanced turbulent approaches often used to solve the fluid dynamic problems. They are Direct Numerical Solution (DNS), Large Eddy Simulation (LES) and RANS-based modeling. The RANS-based modeling is the most frequently used in solving various turbulent flow problems.

In this approach, the turbulent conservation equations are derived by decomposing the NavierStokes equations. All decomposed terms are obtained from the Reynolds decomposition method. The decomposed equations are often known as the Reynolds Average Navier-Stokes (RANS) equations.

There are several RANS turbulent models. They are Spalart Allmaras as one equation turbulent model, $\mathrm{k}-\varepsilon$ and $\mathrm{k}-\omega$ turbulent models as two equation turbulent model and Reynolds Stress 
Model (RSM). Each of the above model has its own variants. The $k-\varepsilon$ model has the variants of the standard $k-\varepsilon$, the RNG $k-\varepsilon$, the Realizable $k-$ $\varepsilon$ and the Low $\mathrm{N}_{\operatorname{Re}} k-\varepsilon$.

The Boussinesq hypothesis is used to close the RANS equations. It is done by introducing the turbulence viscosity, $\mu_{t}$. The RANS equations are written as follows,

$$
\begin{gathered}
\frac{\partial \bar{u}_{x}}{\partial x}+\frac{\partial \bar{u}_{y}}{\partial y}+\frac{\partial \bar{u}_{z}}{\partial z}=0 \\
\rho \frac{\partial \bar{u}_{x}}{\partial t}+\rho \bar{u}_{x} \frac{\partial \bar{u}_{x}}{\partial x}+\rho \bar{u}_{y} \frac{\partial \bar{u}_{x}}{\partial y}+\rho \bar{u}_{z} \frac{\partial \bar{u}_{x}}{\partial z}=-\frac{\partial \bar{p}}{\partial x}+ \\
\frac{\partial}{\partial x}\left(\mu_{e f f} \frac{\partial \bar{u}_{x}}{\partial x}\right)+\frac{\partial}{\partial y}\left(\mu_{e f f} \frac{\partial \bar{u}_{x}}{\partial y}\right)+\frac{\partial}{\partial z}\left(\mu_{e f f} \frac{\partial \bar{u}_{x}}{\partial z}\right)+ \\
\rho g_{x} \\
\rho \frac{\partial \bar{u}_{y}}{\partial t}+\rho \bar{u}_{x} \frac{\partial \bar{u}_{y}}{\partial x}+\rho \bar{u}_{y} \frac{\partial \bar{u}_{y}}{\partial y}+\rho \bar{u}_{z} \frac{\partial \bar{u}_{y}}{\partial z}=-\frac{\partial \bar{p}}{\partial y}+ \\
\frac{\partial}{\partial x}\left(\mu_{e f f} \frac{\partial \bar{u}_{y}}{\partial x}\right)+\frac{\partial}{\partial y}\left(\mu_{e f f} \frac{\partial \bar{u}_{y}}{\partial y}\right)+\frac{\partial}{\partial z}\left(\mu_{e f f} \frac{\partial \bar{u}_{y}}{\partial z}\right)+ \\
\rho g_{y} \\
\rho \frac{\partial \bar{u}_{z}}{\partial t}+\rho \bar{u}_{x} \frac{\partial \bar{u}_{z}}{\partial x}+\rho \bar{u}_{y} \frac{\partial \bar{u}_{z}}{\partial y}+\rho \bar{u}_{z} \frac{\partial \bar{u}_{z}}{\partial z}=-\frac{\partial \bar{p}}{\partial z}+ \\
\frac{\partial}{\partial x}\left(\mu_{e f f} \frac{\partial \bar{u}_{z}}{\partial x}\right)+\frac{\partial}{\partial y}\left(\mu_{e f f} \frac{\partial \bar{u}_{z}}{\partial y}\right)+\frac{\partial}{\partial z}\left(\mu_{e f f} \frac{\partial \bar{u}_{z}}{\partial z}\right)+ \\
\rho g_{z}
\end{gathered}
$$

The standard $k-\varepsilon$ turbulent model is supported by modeling $\mu_{t}$ semi-empirically. The formulation of $\mu_{t}$ variable is given by,

$$
\mu_{t}=C_{\mu} \rho \frac{k^{2}}{\varepsilon}
$$

$k$ is the specific turbulent kinetic energy. $\varepsilon$ is the dissipation rate of the turbulent kinetic energy. $C_{\mu}$ is the empirical constant.

Two new dependent variables, namely the $k$ and $\varepsilon$ variables are modeled by using the conservation equation approach. The concervation equations for these two turbulent variables are expressed as,

$$
\begin{gathered}
\rho \frac{\partial k}{\partial t}+\rho \bar{u}_{x} \frac{\partial k}{\partial x}+\rho \bar{u}_{y} \frac{\partial k}{\partial y}+\rho \bar{u}_{z} \frac{\partial k}{\partial z}= \\
\frac{\partial}{\partial x}\left(\frac{\mu_{e f f}}{\sigma_{k}} \frac{\partial k}{\partial x}\right)+\frac{\partial}{\partial y}\left(\frac{\mu_{e f f}}{\sigma_{k}} \frac{\partial k}{\partial y}\right)+\frac{\partial}{\partial z}\left(\frac{\mu_{e f f}}{\sigma_{k}} \frac{\partial k}{\partial z}\right)+ \\
P_{k}-\rho \varepsilon \\
\rho \frac{\partial \varepsilon}{\partial t}+\rho \bar{u}_{x} \frac{\partial \varepsilon}{\partial x}+\rho \bar{u}_{y} \frac{\partial \varepsilon}{\partial y}+\rho \bar{u}_{z} \frac{\partial \varepsilon}{\partial z}= \\
\frac{\partial}{\partial x}\left(\frac{\mu_{e f f}}{\sigma_{\varepsilon}} \frac{\partial \varepsilon}{\partial x}\right)+\frac{\partial}{\partial y}\left(\frac{\mu_{e f f}}{\sigma_{\varepsilon}} \frac{\partial \varepsilon}{\partial y}\right)+\frac{\partial}{\partial z}\left(\frac{\mu_{e f f}}{\sigma_{\varepsilon}} \frac{\partial \varepsilon}{\partial z}\right)+ \\
C_{\varepsilon 1} \frac{\varepsilon}{k} P_{k}-C_{\varepsilon 2}{ }^{*} \rho \frac{\varepsilon^{2}}{k}
\end{gathered}
$$

There are six dependent variables contained in the $k-\varepsilon$ turbulent model, those are $p, u_{x}, u_{y}, u_{z}, k$ and $\varepsilon$.

Variables $P_{k}$ and $C_{\varepsilon 2}{ }^{*}$ are the turbulent kinetic energy production rate and the constant. These can be found in the literature [12]. The constant values of the equations above are as follows $C_{\mu}=0.085$, $\sigma_{k}=0.7194, \sigma_{\varepsilon}=0.7194, C_{\varepsilon 1}=1.42$ and $C_{\varepsilon 2}=$ 1.68 [12].

These conservation equations can be solved simultaneously using the numerical method. All the numerical procedures for solving all equations are blended in a CFD code.

\section{Research methodology}

This research was conducted using the numerical simulation method with Ansys Fluent as CFD engine. This research is started by designing the burner geometry which includes determining the shape and the size of the burner. The actual designed burner geometry is then built and meshed three dimensionally in the CFD engine.

The CFD simulation is initiated with the problem definitions by assigning all boundary conditions of the specified problem to the meshed boundaries. The next stage is to choose the appropriate turbulent model to simulate the fluid dynamic characteristics in the designed tangential burner. CFD parametric explorations are conducted then to assure that the used CFD parameters produce consistent results. The stage is continued by quantifying the effect of the initial tangential intensity on the fluid dynamic characteristics in the tangential burner using the selected turbulent model.

\subsection{Burner geometry}

The designed tangential burner in this study has single tangential injection. The tangential injection is installed at the bottom of the frustum that connects the axial inlet and the cylindrical body. The frustum length is about $500 \mathrm{~mm}$. The geometry of the tangential burner is shown in Fig. 1.

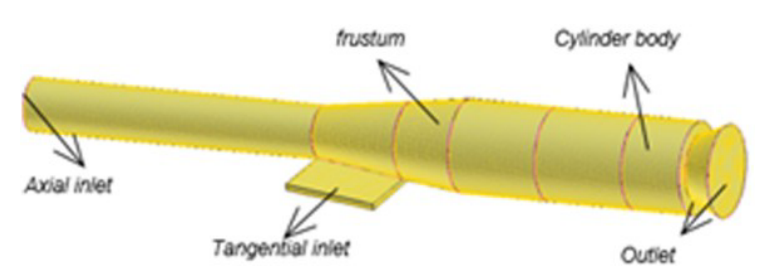

Fig. 1. The geometry of designed tangential burner.

The cylinder diameter is $300 \mathrm{~mm}$ with the length of $900 \mathrm{~mm}$. The axial inlet is $172 \mathrm{~mm}$ in diameter and $1000 \mathrm{~mm}$ in length. The tangential inlet width is $300 \mathrm{~mm}$ and the tangential inlet length is $300 \mathrm{~mm}$. The burner inlet aspect ratio is 14 .

\subsection{Boundary Conditions}

The burner has two inlet, namely axial and tangential inlet, and single outlet. The burner is operated at the atmospheric pressure and isothermal condition. The axial and tangential inlet velocities are varied for several $\mathrm{I}_{\mathrm{TI}}$ values. The variations of $\mathrm{I}_{\mathrm{TI}}$ values are $0.4,0.7,1.1,1.9,3.2,4.5,6.8$, and 10 .

This three dimensional fluid flow simulation is set with the stationary walls with no slip condition. The fluid flows near the walls are governed by the standard wall function. 


\subsection{Model selection}

In this study, the simulations are intended for the design purpose where a very high accuracy of predictions does not always become a primary concern. The three dimensional CFD works with complex physical phenomena require very large computational efforts and need a large computer memory. Such this computer facility is very expensive and rarely available for many engineering design companies. To avoid the problems, it is required to use a proper and robust turbulent model and an inexpensive computational technique for the engineering design purposes.

The DNS approach in solving the turbulent flow produces very high accuracy predictions. Therefore the DNS is intended for the validation purpose rather than for the design purpose. The DNS needs a huge computational effort and an excessive computing time. The LES turbulent model still require a very high computational effort and longer computing time although it has a higher accuracy prediction than the RANS turbulent models.

The RANS turbulent models are able to predict the turbulent flow behaviors appropriately and require a relatively low computational effort. The success of RANS turbulence model in modeling the tangential flow has been widely reported by many authors [6], [8]. But, there is no turbulence model that can work well for all geometries and operating conditions. Therefore, an suitable model for an encountered problem must be chosen carefully.

In addition, the current computers and CFD techniques are well established and mature. These allow the CFD simulations to be carried out using an inexpensive computer facility like a small computing server or a simple Personal Computer (PC) or even a laptop computer with acceptable results. Based on the above considerations, the turbulent models used in this study are the RANSbased turbulent models.

\subsection{Experimental variables}

The numerical quantification on the effect of the initial tangential intensity $\left(\mathrm{I}_{\mathrm{TI}}\right.$ ) to the fluid flow characteristics was conducted in the range from low to high $\mathrm{I}_{\mathrm{TI}}$ values. To eliminate the effect of the other variables, the variables such as the size and shape of the geometry, the tangential inlet slope, the inlet aspect ratio, the position of tangential inlet and mass flow rate are kept constant.

The dependent variables that quantified in this study are the turbulence intensity, the residence time and the pressure drop. These variables are chosen because they have significant effects to the overall burner performances. The turbulence intensity as a degree of the mixing and the residence time have a significant influence on the combustion process, while the pressure drop affects the operating cost of the burner.

\section{Results and discussion}

\subsection{RANS models performance}

The selection of the turbulent model among the RANS turbulent models is based on the ability of the models to agree with the experimental data of Chen et al. [4]. These data are used because they were measured from the chamber which has similar geometry to the tangential burner used in this study. The RANS turbulent models which are evaluated here are standard $k-\varepsilon$, RNG $k-\varepsilon$, Realizable $k-\varepsilon, k-\omega$ and RSM model.

The detailed geometry of the Chen's chamber can be found on the original paper of Chen [4]. The model evaluations are done on the case of the high swirl flow $\left(\mathrm{I}_{\mathrm{TI}}\right.$ value $\left.=0.8\right)$ and with mesh interval size $3.3 \%$ of the burner diameter. This mesh interval size is obtained by conducting the mesh size parametric study. The comparisons between our prediction results and the Chen experimental data are shown in Fig. 2.

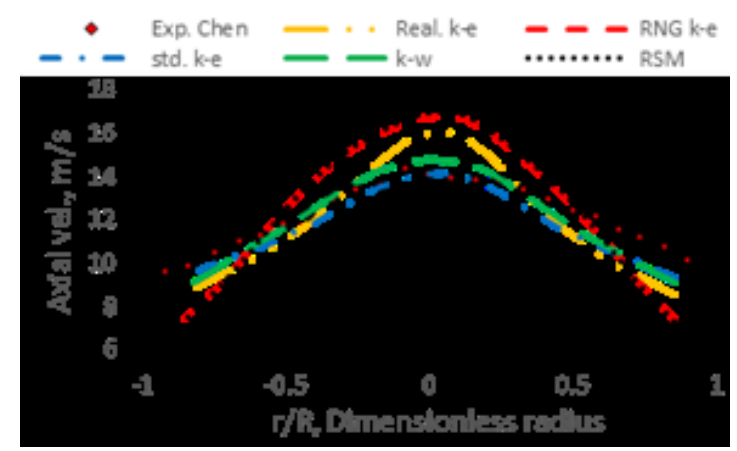

(a) Axial velocity

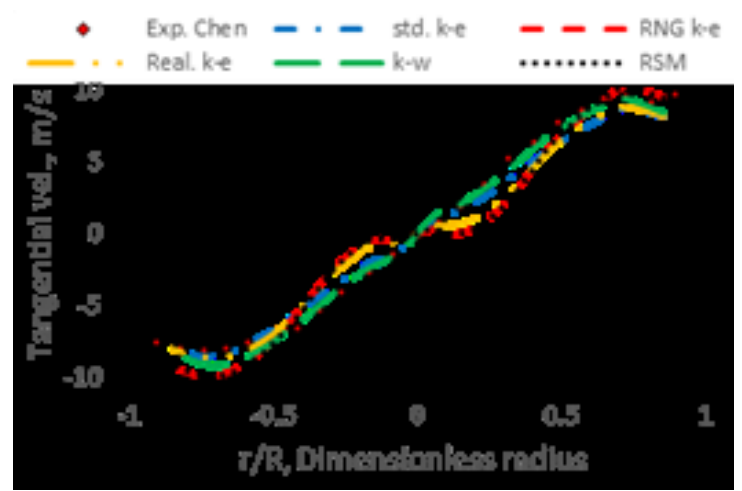

(b) Tangential velocity

Fig. 2. Comparison of some RANS turbulent models performances.

Although with the inherent isotropic turbulent assumption, the $k-\varepsilon$ turbulent models are able to arrive with good velocity predictions for a high swirl flow, especially the standard $k-\varepsilon$ model. These predictions are more accurate than those given by RSM, although theoretically, the RSM model supposed to provide the better results because it was built under the anisotropic turbulent assumption. These are consistent with statement of Vazquez, 
who reported that for an atmospheric swirl burner, the predictions of $k-\varepsilon$ model for axial and tangential velocity are better than RSM [6].

Quantitatively, the prediction results of standard $k$ - $\varepsilon$ model deviate from experimental data about $12 \%$ for the tangential velocity and $5 \%$ for the axial velocity. In addition, the standard $k$ - $\varepsilon$ turbulent model is easy to converge and has relatively low computational effort comparing to other RANS models. Based on the things above, the standard $k-\varepsilon$ turbulent model is chosen to quantify the fluid flow characteristics in this study.

\subsection{Quantification of the effect of $I_{T I}$ value to the fluid dynamic characteristics}

The numerical quantifications in this study were conducted in the tangential burner (Fig. 1) that has $0^{0}$ of the tangential inlet slope, 14 of the inlet aspect ratio and the tangential inlet position at the bottom of the frustum. The simulations were done using the standard $k-\varepsilon$ turbulent model at constant total mass flow rate of $0.24 \mathrm{~kg} / \mathrm{s}$.

\subsubsection{Turbulence intensity}

In this simulation, the values of the mean turbulence intensity of the flow are represented by the mean values of the turbulence intensity of 29 fluid flow tracks. The profiles of the turbulence intensity value for each track was shown in Fig. 3.

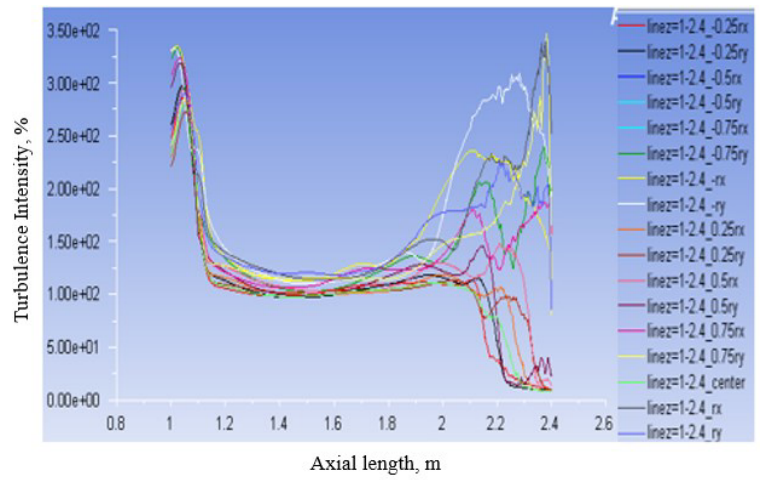

Fig. 3. Turbulence intensity profile along burner at several release points (case for $\mathrm{I}_{\mathrm{TI}}=10$ ).

The turbulence intensity values near the tangential inlet and the outlet zone are greater than other zones along the burner, as shown in Fig. 3. The high value of the turbulence intensity near the tangential inlet zone $(z= \pm 1)$ is caused by the mixing of the axial and tangential inlet flows. The high values of this variable near the outlet $(z= \pm$ 2.4 ) is due to the sudden contraction.

The mean turbulence intensity along the tangential burner is influenced by the $\mathrm{I}_{\mathrm{TI}}$ value. The higher the $\mathrm{I}_{\mathrm{TI}}$ value, the higher the turbulence intensity value is. The profiles of the mean turbulence intensities for several $\mathrm{I}_{\mathrm{TI}}$ values are shown by Fig. 4.

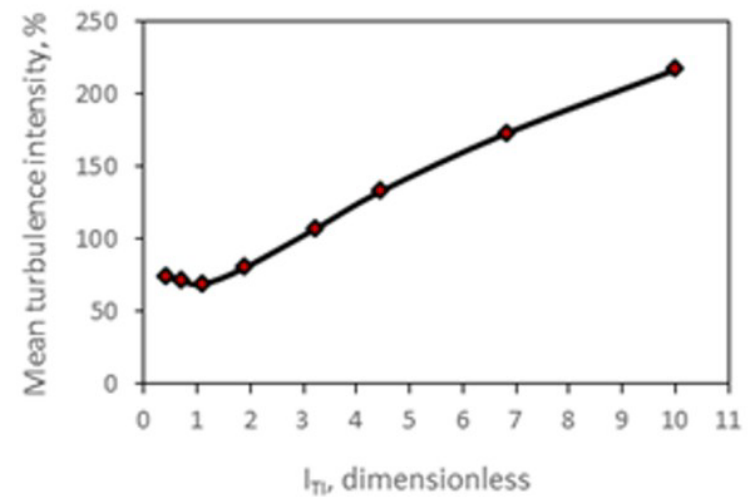

Fig. 4. Mean turbulence intensity profile in a tangential burner for several $\mathrm{I}_{\mathrm{TI}}$ values.

\subsubsection{Residence time}

The air residence times in a burner are computed by dividing the path lengths of the air with the magnitude velocities. The air path length is determined by computing the air pathline formed along the burner. The air pathlines at various release points, either from the axial or the tangential inlet, are shown in Fig. 5. The air pathlines in the figures clearly showed that the flow structures throughout the burner are dominated by the tangential flow.

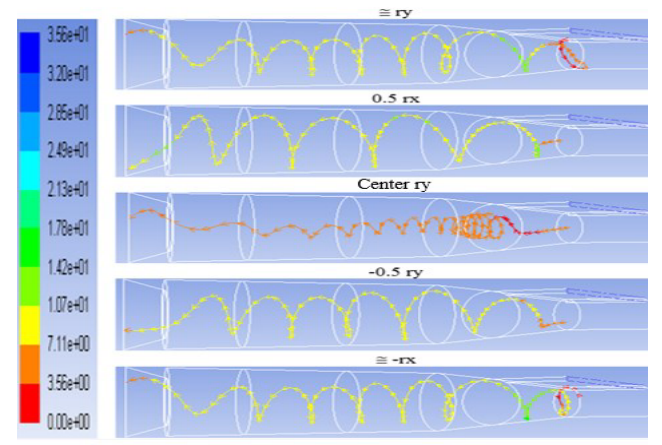

(a) Some release point at axial inlet

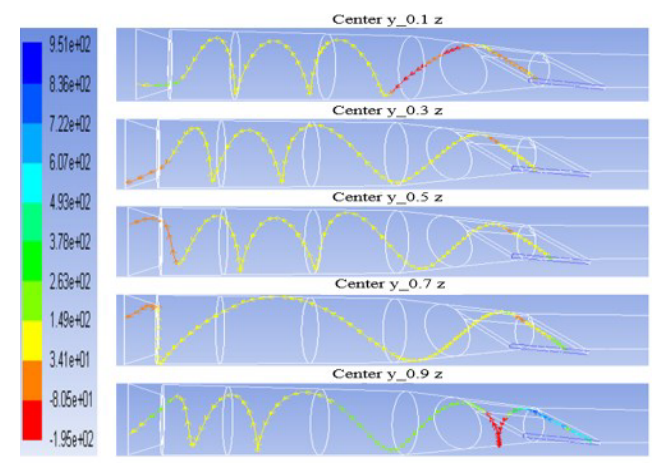

(b) Some release point at tangential inlet

Fig. 5. Air flow pathline for several release points (case for $\left.\mathrm{I}_{\mathrm{TI}}=10\right)$.

A different finding, from those reported by Nemoda about flow structure was obtained in this research. Recirculation flow structure, that is supposed to be produced in high swirl flow, is not 
found during the simulation, even on a very high swirl flow $\left(\mathrm{I}_{\mathrm{TI}}=10\right)$. Whereas according to Nemoda [8], the recirculation flow structure will be formed at $\mathrm{I}_{\mathrm{TI}}$ value $\geq 2.48$. This indicates that the formation of the recirculation flow structure is not only determined by the $\mathrm{I}_{\mathrm{TI}}$ but also determined by the other variables.

The mean residence time along the burner is obtained from the mean air residence time of 20 release points. The profiles of mean residence times in the tangential burner for some of $\mathrm{I}_{\mathrm{TI}}$ values are given in Fig. 6. The mean residence times along the tangential burner are influenced by the $\mathrm{I}_{\mathrm{TI}}$ values. The higher the $\mathrm{I}_{\mathrm{TI}}$ value, the longer the pathline, and the greater the residence time is. Its influence is more significant on the high $\mathrm{I}_{\mathrm{TI}}$ value.

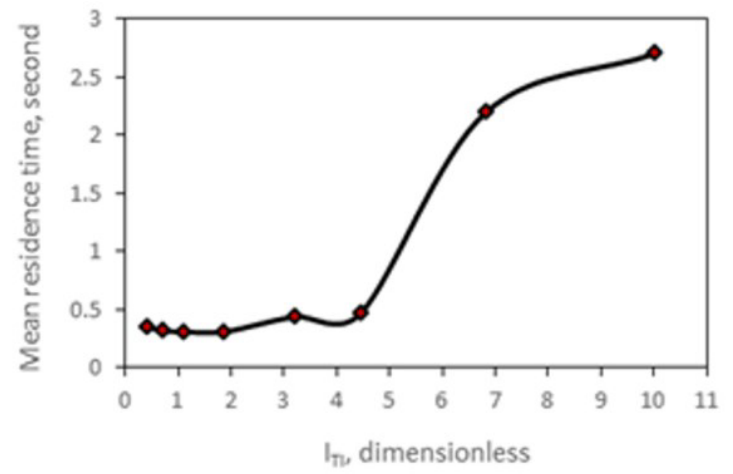

Fig. 6. Mean residence times in a tangential burner for several ITI values.

\subsubsection{Pressure drop}

The mean pressure drop in this study is calculated from the difference between mean static pressure at the burner inlet and the burner outlet. The simulation results showed that the mean pressure drop across the burner is influenced by the $\mathrm{I}_{\mathrm{TI}}$ value. Generally, the greater the $\mathrm{I}_{\mathrm{TI}}$ value, the greater the pressure drop is. This is consistent with the results that are reported by Aydin, where the higher the Reynolds number (it means the higher the $\mathrm{I}_{\mathrm{TI}}$ value), the higher the pressure drop is [11].

Within the range of $\mathrm{I}_{\mathrm{TI}}$ values that is investigated here, the lowest mean pressure drop is produced at the $\mathrm{I}_{\mathrm{TI}}$ value of 3.2. The profiles of the mean pressure drop in the tangential burner at various $\mathrm{I}_{\mathrm{TI}}$ values are given in Fig. 7.

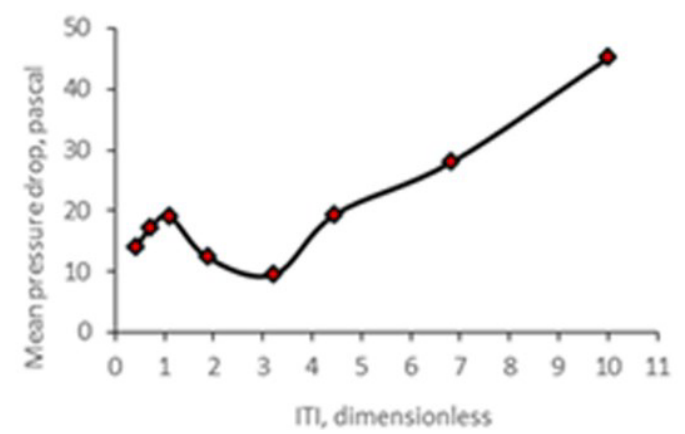

Fig.7. Mean pressure drop in a tangential burner for several $\mathrm{I}_{\mathrm{TI}}$ values.
The effects of the $\mathrm{I}_{\mathrm{TI}}$ on the mean pressure drop are more significant at higher $\mathrm{I}_{\mathrm{TI}}$ value than the lower $\mathrm{I}_{\mathrm{TI}}$ value. This correlates to the pathline (residence time) of the air along the burner where if the pathlines are longer, the pressure loss that is caused by the friction will be greater. The consistency between the residence time and the pressure drop curves can be seen from Fig. 6 and 7 .

\section{Conclusion}

The numerical quantification of the fluid dynamic characteristics in a tangential burner has been carried out using the standard $k-\varepsilon$ turbulent model. The simulation results concluded that three dimensional flow structures in a tangential burner are well described as the dominant tangential flows. The numerical quantifications revealed that the fluid dynamic characteristics are greatly affected by the initial tangential intensity.

In general, the higher the $\mathrm{I}_{\mathrm{TI}}$ value, the greater the mean turbulence intensity, the longer the mean residence time and the higher the mean pressure drop are. At low $\mathrm{I}_{\mathrm{TI}}$ values, the effect of $\mathrm{I}_{\mathrm{TI}}$ on the mean turbulence intensity and the mean residence time are insignificant. The effect of $\mathrm{I}_{\mathrm{TI}}$ on the mean turbulence intensity begin to be significant at the $\mathrm{I}_{\mathrm{TI}}$ values $\geq 1.1$, while its effect on the mean residence time is significant at the $I_{T I}$ values $\geq 4.5$. The lowest mean pressure drop in this study is found at $\mathrm{I}_{\mathrm{TI}}$ value of 3.2 .

Our thanks go to the Ministry of Research, Technology and Higher Education of the Republic of Indonesia for the funding support provided to the implementation of this research through the Decentralization Research Scheme.

\section{References}

1. S. Paulrud, C. Nilsson, Fuel, 83, 813-821 (2004)

2. S. M. B. Kops, P. C. Malte, Department of Mechanical Engineering University of Washington, 149 (2004)

3. J. H. S. Arnao, D. J. O. Ferreira, C. G. Santos, J. E. Alvarez, L. P. Rangel, S. W. Park, Industrial, Mechatronic and Manufacturing Engineering, 9, 798$801(2015)$

4. J. Chen, B. S. Haynes, D. F. A. Fletcher, Melbourne, Australia, 485-490 (1999)

5. J. F. Bourgouin, J. Moeck, D. Durox, T. Schuller, S. Candel, C.R. Mecanique, 341, 211-219 (2013)

6. J. A. R. Vazquez, Thesis, University of Zaragoza, Spain, 1-192 (2012)

7. A. Escue and J. Cui, Appl. Math. Model, 34, 28402849 (2010)

8. S. Nemoda, V. Bakic, S. Oka, G. Zivkovic, N. Crnomarkavic, Int. J. Heat Mass Tran., 48, 4623-4632 (2005)

9. Z. Li, J. Jing, G Liu, Z. Chen, Chem. Eng. Sci., 65, 1253-1260 (2010)

10. M. Momeni, C. Yin, S. K. Kær, T. B. Hansen, P. A. Jensen, P. Glarborg, Energ Fuel, 27, 507-514 (2013)

11. Anysy Inc., Ansys Documentation, Anysy Inc., Canonsburg, PA, United States, (2013) 